\section{An improved BASIC program to produce random samples without replacement}

\section{RICHARD S. LEHMAN}

Franklin and Marshall College, Lancaster, Pennsylvania 17604

Robertson (1977) presented an algorithm for sampling $\mathrm{N}$ values from an array of numbers, $1,2, \ldots$, $M$, without replacement. His procedure is a refinement of that presented by Scheirer (1976), yet it too is less than optimally efficient.

To illustrate the difficulty, consider Robertson's example. He starts with an array X(I) as follows:

$$
\begin{array}{cccccccccc}
\text { Storage Locations: } & X(0) & X(1) & X(2) & X(3) & X(4) & X(5) & X(6) & X(7) \\
\text { Contents: } & 1 & 2 & 3 & 4 & 5 & 6 & 7 & 8
\end{array}
$$

Selecting an element from $\mathrm{X}(\mathrm{I})$ is accomplished by generating a random number in the range 0.7 (e.g., 3 ) and using the contents of X(3) as the output.

Robertson's algorithm next has one delete X(3) from the array by shifting all elements above X(3) left one location, so that the array appears as:

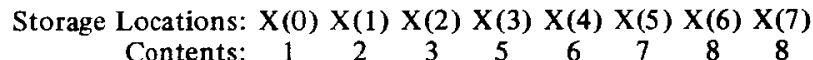

The next random value is selected by generating a value in the range $0-6$, since the value in $X(7)$ is no longer needed.

The inefficiency in this algorithm results from having to move all values from $X(I) \ldots X(K)$, where $I$ is the just-selected value and $K$ is the last useful entry. $A$ much simpler approach is to simply move the last (Kth) value into the just-selected (Ith) location. The number of entries in the array is then decreased by 1 and the algorithm continues. Following this procedure, the array would have this appearance after $\mathrm{X}(3)$ was selected:

$$
\begin{array}{lllllllll}
\text { Storage Locations: } & X(0) & X(1) & X(2) & X(3) & X(4) & X(5) & X(6) & X(7) \\
\text { Contents: } & 1 & 2 & 3 & 8 & 5 & 6 & 7 & 8
\end{array}
$$

The next value is selected by generating a value in the range $0-6$, as previously. Table 1 presents the modified version of Robertson's program. Note that the contents if $X(I)$ are no longer ordered sequentially, but that is of no consequence in the algorithm.

To illustrate the relative efficiency of this modified algorithm, a series of random samples was generated using the two programs. Table 2 presents the mean time (in CPU seconds for 10 replications) to generate the samples described. For example, in generating samples of size $\mathrm{N}=5$ from the range of integers $1-10$, Robertson's program required an average of $.03 \mathrm{sec}$, while the revision presented here needed $.02 \mathrm{sec}$. As the values of $N$ and $M$ increase, the inefficiency becomes even more pronounced. (The timing reported here is for BASIC on a Univac 70/46 under VMOS; actual times will vary by computer, but the ratio of one algorithm to the other should remain relatively stable across computer systems.)

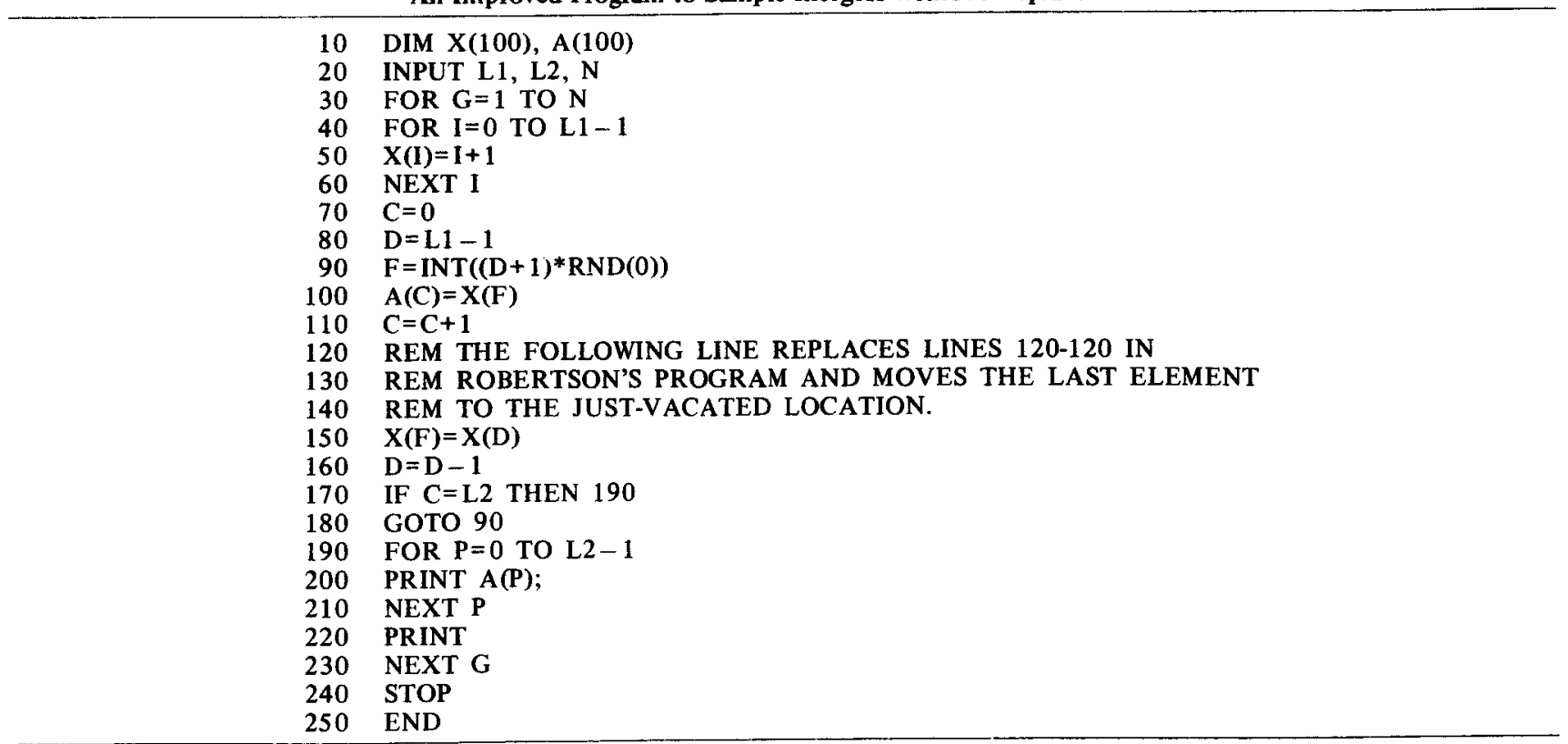


Table 2

Mean Times (CPU Seconds) to Generate Samples of Size $\mathbf{N}$ from a Population Consisting of the Values 1, 2, . , M

\section{Algorithm}

\begin{tabular}{rrrr} 
N & M & Original & Improved \\
\cline { 3 - 4 } 3 & 5 & .01 & .01 \\
5 & 5 & .02 & .02 \\
5 & 10 & .03 & .02 \\
10 & 10 & .04 & .03 \\
100 & 100 & 1.77 & .27 \\
10 & 1000 & 3.80 & .55 \\
100 & 1000 & 30.65 & .74 \\
1000 & 1000 & 173.79 & 2.72 \\
\hline
\end{tabular}

The present procedure is one of a set of four (equally likely elements, with and without replacement, and unequal probabilities, with and without replacement) that encompass the majority of sampling situations of this nature. Algorithms for all four sampling problems are presented by Lehman (1977).

\section{REFERENCES}

Lehman, R. S. Computer simulation and modeling: An introduction. Hillsdale, N.J: Lawrence Erlbaum, 1977.

Robertson, S. A. A BASIC program to produce random samples without replacement. Behavior Research Methods \& Instrumentation, 1977, 9, 363-364.

SCHEIRER, C. J. Routine to provide a random order for counterbalanced variables. Behavior Research Methods \& Instrumentation, 1976, 8, 31.

(Accepted September 28, 1977.) 